\title{
Ethiopian Economic Features and Changing Persistence: A Time Series Analysis
}

\author{
Tofik Mussa Reshid \\ Department of Statistics, Werabe University, Werabe, Ethiopia
}

Email address:

toffamr@gmail.com

\section{To cite this article:}

Tofik Mussa Reshid. Ethiopian Economic Features and Changing Persistence: A Time Series Analysis. American Journal of Theoretical and Applied Statistics. Vol. 9, No. 3, 2020, pp. 37-46. doi: 10.11648/j.ajtas.20200903.12

Received: October 15, 2019; Accepted: April 15, 2020; Published: April 28, 2020

\begin{abstract}
Ethiopia was one of the countries least developed and it is among the countries in the bottom in the rank of GDP's that UN lists. However, nowadays Ethiopia is one of the fastest growing economies in the world. In Ethiopia much effort has been made to build the national economy. Ethiopia has made significant strides towards becoming a middle income country by 2025. This paper provides an overview of Box-Jenkins model for temporal data. In this research we used time series analysis of some of Ethiopian economic features such as GDP, GDP growth rate and inflation rate. Box-Jenkins model was used to analyze 35-year data (1981-2015). GDP, GDP growth rate, and inflation rate were variables under the study to describe persistence change and to forecast future behaviors. We tried to find best model for description and predictive model for these series using different model selection tools. We compared different orders of Autoregressive Integrated Moving Average (ARIMA) using AIC, BIC and MSE to fit the observed data. The best from compared was ARIMA $(2,2,2)$ for GDP, ARIMA $(2,1,2)$ for GDP growth rate and ARIMA $(1,1,1)$ for inflation rate. Since forecasting is important for many purposes, we forecast the series from best ARIMA models. Five year forecast showing that GDP is an increasing trend and the average forecast of GDP rates is showing an average of 10.028 .
\end{abstract}

Keywords: ACF, PACF, ARIMA, ARMA, Inflation, GDP, Forecast, Box-Jenkins, Box-Ljung

\section{Introduction}

\subsection{Background of the Study}

The major aim of countries is to build strong economy. In Ethiopia much effort has been made to build the national economy. Ethiopia has made significant strides towards becoming a middle income country by 2025 [1]. The Ethiopian economy is undergoing rapid transformation and has become the fastest growing non-oil exporting economy in the world, posting an average of $11 \%$ growth annually over the last eight years [1]

One of the tools used to measure the economic development of a given nation is the GDP of the nation. Ethiopia was one of the countries least developed. The human development index (HDI) calculated yearly by UN ranks Ethiopia 169th from 177 countries in 2005 and its GDP percapita was of \$ US 160 . But during last decades it has become one of the fastest growing economies in the world with an average GDP growth rate $10 \%$ per annum [21]. In contrary to the previous three or four decades starting from 1990s the economy is in its fast growth trend [22].

To overcome poverty and for sustainable development the government formulated the five year growth and transformation policy (GTP). On the level of economic development, the country has taken remarkable steps towards market based reform such as trade regulation privatization and agricultural led industrialization. That makes Ethiopia one of the fastest growing economy in the world [23]. However, the approach was not very successful in increasing industrial output and food productivity [6]

In this research time series analysis was used to describe the Ethiopian economic characteristics. Changing persistent has been an important features economic time series such as inflation (Barsky, 1987 [9], Burdekin and Siklos, 1999 [12]), interest rate (Mnkiw, et al. 1987 [13]), and government deficit's (Hakio [14]) Box and Jenkins developed a model for stationary time series data $[4,7]$. This model is called autoregressive integrated moving average (ARIMA). It is well known forecasting model for a time series [20]. The model has been 
applied in different disciplines such as industry, economics and medical researches $[8,10,11]$. This model enables us to assess the trend, to describe the characteristics, to simulate and forecast the future behavior of the series. In this research ARIMA model was applied on thirty five year data of Ethiopian GDP, GDP growth rate and inflation rate (called GDP deflator). The goodness of the model can be assessed using different approaches. In our case we use autocorrelation function (ACF) and partial autocorrelation function (PACF) plot to identify the order of ARIMA. The most powerful test is portmanteau statistics of Box-Ljungs test of ARMA (p, q) model diagnosis.

\subsection{Statement of the Problem}

One of the major areas of concern of time series is economics. In this study we used Box-Jenkins model which concerned with the relationship between observations oriented in time sequence. If a proper time series model is built it can describe the features of the series and it is powerful forecasting tool for macro and micro of economics.

There are only few researches in Ethiopian economy done using in time series model. Our goal here was to build explaining model for Ethiopian economic characteristics and to asses GDP, GDP growth rate and Inflation. This research will motivate to study more on the area in addition to it inspire to answer the following questions.

a. What is underlying characteristics of Ethiopian GDP, GDP rate and Inflation

b. How to assess the progress of the Ethiopian economy?

c. How to project future economies?

\subsection{Significance of the Research}

Statistical analysis has diverse applications in several disciplines. The outputs of the research were provided model for characteristics feature of Ethiopian GDP, GDP growth rate and inflation rate. Time series analysis of Ethiopian GDP, GDP growth rate and inflation rate in stochastic process was analyzed. The analysis of the research method can then be integrated in to economics to identify the trend of Ethiopian economy as well as the analysis is used how to handle stochastic data in econometric analysis. In addition to the study gives recent information.

Moreover, there has been an increasing interest in modeling. In the study theories and computer algorithms were used. Therefore, the importance of this study may serve as basis for further research analysis and it will have paramount importance for theoretical frame work and practices.

\subsection{Objectives}

The genera objective of this study was to assess the trend of Ethiopian economic characteristics and to build explaining model. And the following points were the specific objectives of the study

a. to assess the growth of Ethiopian GDP

b. to assess the growth rate of GDP

c. to develop ARIMA model which fits to the data

d. to project future characteristics and growth

\section{Materials and Methods}

\subsection{Study Area}

The study was carried out in Ethiopia. Ethiopia is a country found in the Horn of Africa. It is the second most populous country in sub-Saharan Africa. Astronomically it roughly lies between $9.4969^{\circ}$ North latitude and $36.8961^{\circ}$ East longitudes. The total area of the country is 1.1 million sq. $\mathrm{km}$. The capital city of Ethiopia is Addis Ababa, which is also the seat of many international organizations. Ethiopia is divided in to 9 regions and two cities administrations.

According to world fact book 2014 report the projection of 2015 growth rate of Ethiopia is $2.89 \%$ and the total population of the nation is estimated $96,633,458$ peoples.

In this research we tried to analyze and model the development of Ethiopian economy features such as GDP, GDP growth rate and Inflation rates. We used Box-Jenkins method of analysis. The data used for this study was period from 19812015. The data was obtained from website of World Bank.

\subsection{Methodology}

Box-Jenkins method for time series analysis is used for stochastic process to analyze observations indexed in time. Let $\left\{Y_{t}: t=1,2, \ldots n\right\}$ be a random variable observed in time $t$ then this variable is called time series variable. The aim is to find a model to describe the features of the series and forecast the future values. Box and Jenkins developed a model for such type of stochastic process. This model is called ARMA which requires stationary time series variables.

Stationarity

There are two types of Stationarity [17]

1. Weak Stationarity (second order stationary): For time series variable $Y_{t}$ weakly stationary is constant in mean and variance and the covariance between two observations at different lag is depends on only the time lag between two observations. A time series variable $Y_{t}$ is weakly stationary if

a. $E\left(Y_{t}\right)=\mu$

b. $\operatorname{Var}\left(Y_{t}\right)=\sigma^{2}$

c. $\operatorname{Cov}\left(Y_{t}, Y_{t-h}\right)=\Gamma(h)$

2. Strictly stationary (first order stationary): if the distribution of the series is normally distributed then it is strongly stationary. In other words, the distribution of the process is invariant under time lag shift [16]

$\left(Y_{t 1} \ldots . Y_{t n}\right)^{D}=\left(Y_{t+h}, \ldots . Y_{t n+h}\right)$ i.e. Equality in distribution

This is strong assumption rarely achieved. For ARMA process at least second order Stationarity is required. BoxJenkins method requires stationary time series data. To assess the Stationarity of the data we have different ways to use. In this research we used time series plot and autocorrelation plots. If the data is non stationary we need to transform in to Stationarity depending on the cause of it. Thus we have to know the source of non Stationarity. There are five sources of non stationarity [17], outliers, random walk, drift, trend and 
changing variance. If non-Stationarity is detected, then we have to transform in to Stationarity by detrending (removing the trend) or differencing. One way of testing non stationarity is evaluating regression of the time series variable on time nesting the mean and differencing. The trend of time series variable is polynomial of function of time. For time series variable $\left\{Y_{t}: t=1,2, \ldots n\right\}$ the first difference is

$$
\nabla Y_{t}=Y_{t}-Y_{t-1}
$$

and we use shift operator $B$ such that

$$
Y_{t-1}=B Y_{t}
$$

Thus

$$
\nabla Y_{t}=(1-B) Y_{t}
$$

The $d^{\text {th }}$ difference is applying first difference $d$ times and using shift operator

$$
\nabla^{d} Y_{t}=(1-B)^{d} Y t
$$

If non stationary time series variable $\left\{Y_{t}: t=1,2, \ldots n\right\}$ is transformed to Stationarity due to $d^{\text {th }}$ difference and if the difference is $\operatorname{ARMA}(p, q)$ then $\left\{Y_{t}: t=1,2, \ldots n\right\}$ is $\operatorname{ARIMA}(p, d$, q) process

ARIMA models

In time series data the underlying process has different types of structures. The adjacent variables are related to one other in different relations. One way involves shock, innovation, or error driving the time order stochastic process [17]. In this case the time series variable is determined by the previous $q$ uncorrelated errors. This model is called moving average order $q$, denoted by $M A(q)$

$$
Y_{t}=e_{t}+\theta_{1} e_{t-1}+\ldots . \theta_{q} e_{t-q}
$$

We can write as lag operator. That is a function of $B$

$$
Y_{t}=\Theta(B) e t
$$

Where $\Theta(B)=\theta_{1} B+\ldots \theta_{q} B^{q}$ and $e_{t}$ error; its distributed is $N\left(0, \sigma^{2}\right)$

Sometimes the observations have some correlation with $\mathrm{p}$ previous observations. In this case autoregressive model of order $p$ is suggested $A R(p)$.

$$
Y_{t}=\phi_{1} Y_{t-1}+\ldots . . \phi_{p} Y_{t-p}+e_{t}
$$

We can write as lag operator. That is a function of B

$$
\Phi(B) Y_{t}=e_{t}
$$

Where $\Phi(B)=\phi_{1} B+\ldots \phi_{p} B^{p}$

Another relation in time series is the process is the combination of $M A(q)$ and $A R(p)$ in this case the series is $\operatorname{ARMA}(p, q)$ is given by:

$$
\Phi(B) Y_{t}=\Theta(B) e t
$$

Where $\Phi(B)$ and $\Theta(B)$ are as defined above

If the series is nonstationary and it transformed to stationary through $d^{\text {th }}$ difference the model is $\operatorname{ARIMA}(p, d, q)$ and it is given by

$$
\Phi(B) \nabla^{d} Y_{t}=\Theta(B) e t
$$

Where $\Phi(B)$ and $\Theta(B)$ are as defined above

If the time series variable is not stationary Box-Jenkins method is not feasible. If the series is stationary there is an indication from parameters of $A R$ and $M A$ process. The parameters are bounded in some interval.

Indication of Stationarity

Stationarity of $A R$ process is such that: if the parameters of the AR process are bounded and it can be converted to infinite order of $M A$ called bound of Stationarity. If the $M A$ parameters are bounded the processes can be converted to infinite order of AR called invertible

Parameter estimation

To estimate parameters of ARIMA process we need ACF and PACF of the series are important. The ACF of the series at lag $h$ is:

$$
\operatorname{Corr}\left(Y_{t}, Y_{t-h}\right)=\frac{\operatorname{Cov}\left(Y_{t}, Y_{t-h}\right)}{\sqrt{\operatorname{Var}\left(Y_{t}\right), \operatorname{Var}\left(Y_{t-h}\right)}}=\frac{\Gamma(h)}{\Gamma(0)}
$$

Where

$$
\operatorname{Cov}\left(Y_{t}, Y_{t-h}\right)=\Gamma(h)=\sum_{t=1}^{n-h}\left(Y_{t}-\bar{Y}\right)\left(Y_{t-h}-\bar{Y}\right) / n-h
$$

The PACF is obtained using recursive formula. The $k^{\text {th }}$ PACF is

$$
\begin{gathered}
\phi_{k k}=\frac{\rho(k)-\sum_{j=1}^{k-1} \phi_{k-1, j} \rho(k-j)}{1-\sum_{j=1}^{k} \phi_{k-1, j} \rho(j)} \\
\phi_{k, j}=\phi_{k-1, j}-\phi_{k k} \phi_{k-1, k-j}, \quad j=1, \ldots . . k-1
\end{gathered}
$$

We fitted the model by estimating parameters of different orders of ARIMA.

Different order of ARIMA models were compared using Information criteria and residual analysis.

Once the parameters are estimated the "best" model can be selected using minimum value of AIC or/and BIC. The Akakie information criteria is

$$
A I C=-2 \log (l)+\frac{2(p+q+1)}{n-p-q-2} n
$$

And Bayesian information criteria is

$$
B I C=-2 \log (l)+\frac{p+q}{n} \log (n)
$$

Where $l$ is the likelihood function 
Goodness of fit

The common statistic for goodness of the model is $\mathrm{R}^{2}[17]$.

$$
R^{2}=\frac{S S R}{S S T}=1-\frac{S S E}{S S T}
$$

Where SSR is sum square fitted, SST is sum square total and SSE is sum square errors.

This statistic is important for goodness of fit, but it does not consider the number of parameters involved in the model. For this case we use adjusted $R^{2}$

$$
\operatorname{Adj}-R^{2}=1-\frac{\operatorname{Var}\left(e_{t}\right)}{\operatorname{Var}\left(Y_{t}\right)}
$$

The other statistic used for goodness of fit is using estimation accuracy of the model.

Mean absolute error.

The difference between the actual series and the values estimated in fitted model in the same time.

$$
M A E=\frac{\sum_{t=1}^{n}\left|e_{t}\right|}{n}
$$

The statistic mean square error is also used

$$
M S E=\frac{\sum_{t=1}^{n} e^{2}{ }_{t}}{n-k}
$$

Where $\mathrm{k}$ is the number of parameters in $\operatorname{ARIMA~}(\mathrm{p}, \mathrm{d}, \mathrm{q})$

We can measure the goodness of the model using residual analysis. The goodness of the model is assessed using portmanteau statistics of Box-Jung test [4, 7] and Box-Pierce test. Residuals are assumed to be identically independently distributed. Portmanteau statistic using Box-Pierce test is used for such purpose.

$$
Q=n \sum_{h=1}^{m} \rho^{2}(h)
$$

We are assumed our model is $\operatorname{ARIMA}(p, d, q)$,

If the null hypothesis is true the statistic follows $\chi^{2}(m-q-p)$. If $\mathrm{Q}$ is smaller than this value our model is correct. i.e. the model is $\operatorname{ARMA}(\mathrm{p}, \mathrm{q})$

Latter Box-Ljung reform Portmanteau statistic and it is more powerful than the Box-Pierce

$$
Q=n \frac{(n+2)}{n-k} \sum_{h=1}^{m} \rho^{2}(h)
$$

\section{Forecast}

There are several methods of forecasting such as regression analysis classical decomposition and Box-Jenkins methods [2]. In this research we applied time series analysis to describe the feature and to predict future values of GDP, GDP rate and inflation rate. If we have observations $Y_{1}, \ldots . Y_{n}$ of time series variable the aim is to predict $Y_{n+1}, Y_{n+2} \ldots$, based on the given information. The prediction can be done minimizing prediction error. The $h$ ahead forecast can be done so that

$$
\operatorname{Min}\left[E\left(Y_{n+h}-\hat{Y}_{n+h}\right)^{2}\right] \text { Where } \hat{Y}_{n+h} \text { is obtained some }
$$

function of the observed series $\hat{Y}_{n+h}=g\left(Y_{1}, \ldots . Y_{n}\right)$

\section{Result and Discussion}

The aim of this research was to assess and forecast the underlying series of Ethiopian economic characteristics, such as annual GDP, GDP growth rate and inflation rate. We use 35 years of data obtained from World Bank. To assess the trend and to forecast the future characteristics of these series we use Box-Jenkins methodology for these time series data analysis. The methodology called Auto Regressive Moving average (ARMA) requires assumptions of Stationary time series observations in discrete time. For analysis and computation, we use R-statistical software [2, 5, 18]. By using the available data, we forecast the coming five years of these economic characters. A 35 year data sets of GDP in billions of USD, GDP rate and inflation rate in Ethiopia has been presented in Table 1 hereunder

Table 1. Annual GDP, GDP rate and inflation rate of Ethiopia (1981-2015).

\begin{tabular}{llll}
\hline year & GDP (billionsUSD) & GDP\%growth & Inflation \\
\hline 1981 & 7.325 & & \\
1982 & 7.708 & 0.916 & 4.273 \\
1983 & 8.568 & 8.235 & 2.703 \\
1984 & 8.096 & -2.848 & -2.734 \\
1985 & 9.481 & -11.144 & 31.788 \\
1986 & 9.849 & 9.662 & -5.273 \\
1987 & 10.527 & 13.859 & -6.119 \\
1988 & 10.909 & 0.504 & 3.106 \\
1989 & 11.477 & -0.361 & 5.585 \\
1990 & 12.175 & 2.726 & 3.271 \\
1991 & 13.464 & -7.137 & 19.084 \\
1992 & 10.493 & -8.672 & 15.532 \\
1993 & 8.831 & 13.143 & 13.377 \\
1994 & 6.928 & 3.19 & 2.931 \\
1995 & 7.664 & 6.128 & 12.706 \\
1996 & 8.548 & 12.426 & 0.239 \\
1997 & 8.589 & 3.134 & 0.207 \\
1998 & 7.818 & -3.458 & -0.15 \\
1999 & 7.701 & 5.162 & 2.223 \\
2000 & 8.242 & 6.073 & 9.465 \\
2001 & 8.231 & 8.301 & -5.755 \\
2002 & 7.851 & 1.515 & -3.621 \\
2003 & 8.624 & -2.161 & 12.768 \\
2004 & 10.131 & 13.573 & 3.911 \\
2005 & 12.401 & 11.819 & 9.876 \\
2006 & 15.281 & 10.835 & 11.552 \\
2007 & 19.708 & 11.456 & 17.221 \\
2008 & 27.067 & 10.789 & 30.312 \\
2009 & 32.437 & 8.803 & 24.146 \\
2010 & 29.934 & 12.551 & 1.445 \\
2011 & 31.953 & 11.178 & 20.062 \\
2012 & 43.311 & 8.648 & 33.541 \\
2013 & 47.648 & 10.582 & \\
2014 & 55.612 & 9.608 & \\
2015 & 61.537 & & \\
\hline & & & \\
\hline
\end{tabular}

Source: World Bank data base.

Data.worldbank.org/NY.GDP.MKT.CD?locations=ET 
According to the data in Table 1 GDP seems increasing trend. Changing persistence has been an important feature of economic time series (Perron, 2006) [3]. The GDP growth rate fluctuated very much in the first decade and half decade of this study time, reached minimum of -11.44 in the year 1985 and maximum of 13.859 in the year 1987. However, after this period it becomes a little beat sustain and less fluctuates. The trend of growth of real GDP can be considered as sustainable economic growth while the shortrun fluctuations of growth over the trend can be thought of as business cycles [10]. The average GDP growth rate for (1981-2015) is 5.57 the average GD growth rate for (20002015 ) is 9.25 and the average GDP growth rate for (20052015 ) is 10.47 and the last five-year average was 10.48 . The growth of the economy was quite impressive with an average growth rate of about 9 percent per annum since 2000 [19]. The Ethiopian economy has experienced impressive growth performance over the last decade with average GDP growth rate of $11 \%$ [10]. Except for the last five years where inflation, owing to lack of prudent monetary and fiscal policy, was a major problem [9]

The inflation fluctuates especially it increases after 2003. Many are unable to sustain themselves, especially following the rampant inflation that began in 2005 [19].

The aim is to find a model to forecast and explain the future behavior of these series. In analysis of time series data, the preliminary step is to present the data graphically for visual aid [2]. Graphical presentation is used to assess Stationarity, which is the fundamental assumption of BoxJenkins models [4]. Stationarity is the basic assumption of ARIMA model we are looking for. Graphs used to assess Stationarity and indicate type of trend and/or pattern of the data. Figure 1 present's 35-year GDP of Ethiopia from 19812015 (a), Annual GDP growth rate 1982-2015 (b) and annual inflation rate 1982-2015 (c)



(a)

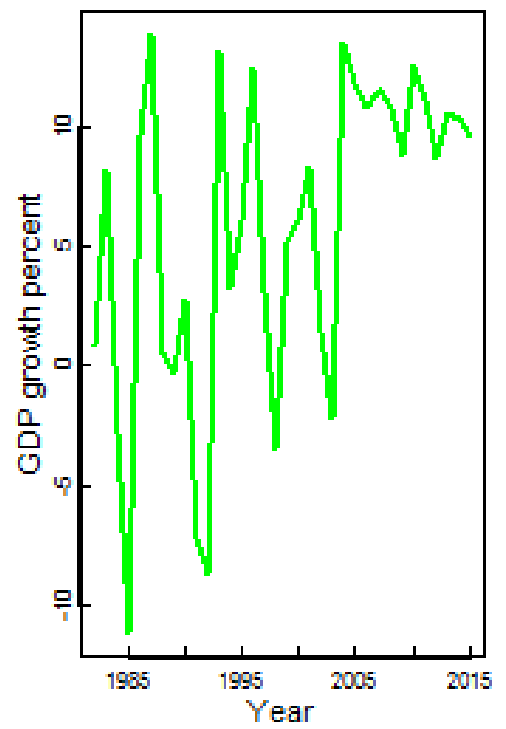

(b)



(c)

Figure 1. (a) the GDP of Ethiopia (b) GDP growth rate and (c) inflation rate (GDP deflator).

The country GDP was showing an increasing trend. The GDP rate fluctuates in previous decades and now it is stabilized. The inflation rate fluctuates it reach its maximum 33.154 in the year 2012. We want to apply Box-Jenkins model for these series that requires stationary series observations. The series looking at Figure 1 (b) is random walk with mean 5.57 and variance 46.5 and the series in (c) are stationary by transformation, such as detrending, differencing or Box Cox transformation.

Statistical test of assessing Stationarity is by using ACF and PACF plots. If the data is stationary in mean the ACF and PACF graph quickly drops to zero. To assess the type of transformation needed, to asses Stationarity of the data and also to determine the ARIMA order we use autocorrelation and partial autocorrelation of the series. Figure 2 looking below is the ACF and PCF against lag is present.

$\mathrm{ACF}$ and PACF drops quickly to zero for stationary series.
Using autocorrelation and partial autocorrelation we can find the order of ARIMA and detect non-Stationarity.

The ACF for GDP Figure 2 (a) is gradual to drops to zero and there is correlation of the observed series in the first few lags, this shows the series is not stationary. The series transformed to Stationarity by detrending or differencing. However, GDP growth rate and Inflation rates (b) and (c) looks stationary. Non-zero ACF lag suggests the order of MA and non-zero PACF Lag suggests AR order. GDP growth rate Figure 2 (b) shows there is no lag having significance ACF and figure 2 (e) there is significant PACF at lag 3 For GDP we take differencing and polynomial trends to transform the series to stationary. To transform in to Stationarity differencing could bring Stationarity and also trend does. For $k^{\text {th }}$ degree polynomial trend, the $k^{\text {th }}$ differencing stabilizes the variance of the series. 


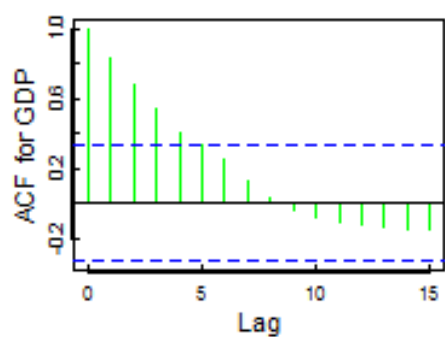

(a)

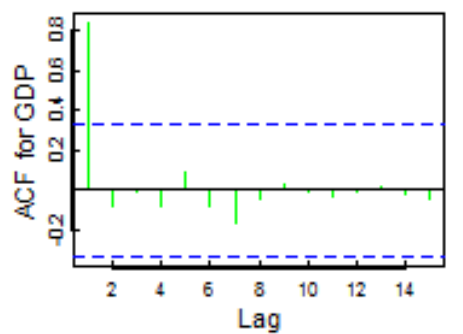

(d)

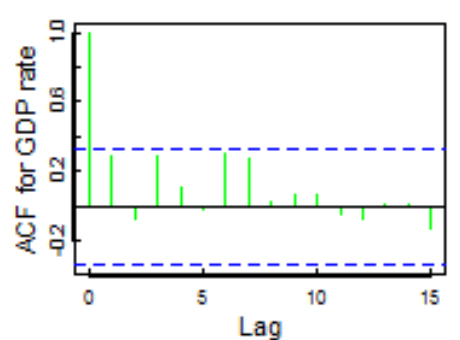

(b)

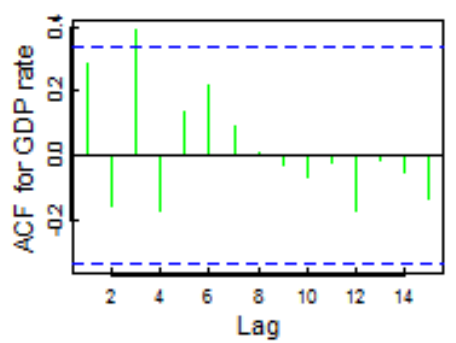

(e)

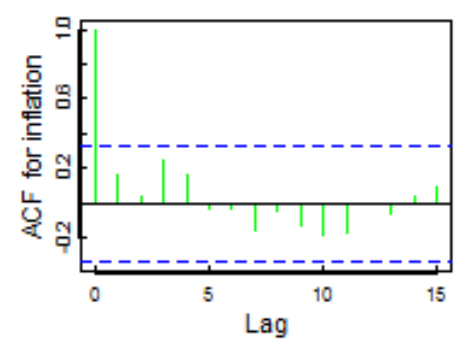

(c)

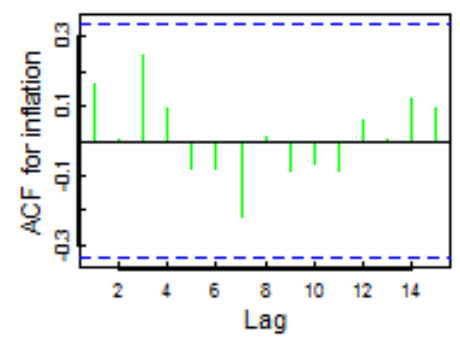

(f)

Figure 2. The autocorrelation and partial autocorrelation of the data.

To see the degree of polynomial fits the series we compare the first three-degree polynomials of time. The third-degree polynomial fit is very similar to the observed series. The data and the fitted trend of third-degree polynomial are looking at figure 3.

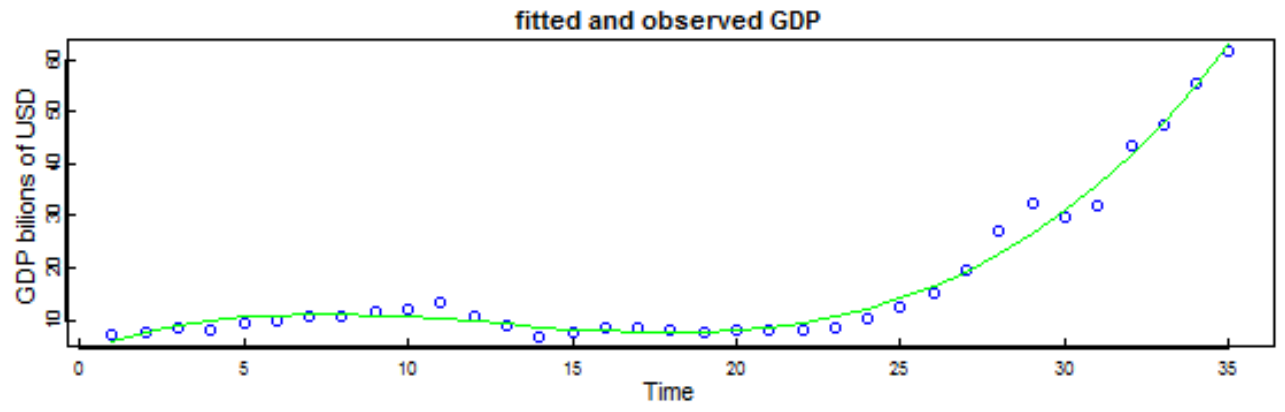

Figure 3. Regression fitted and observed series of GDP.

The observed GDP was fitted using third degree polynomial. The OLS estimation is used for fitting the trend. Multiple $\mathrm{R}^{2}$ of the model is 0.9839 and the residual standard error is 1.96 with 31 degrees of freedom. The observed data is very similar to the fitted series. We also take the third difference to see whether it achieve Stationarity. The third difference and residuals from third degree polynomial are taken as time series observations. We present these series at Figure 4 and also it is helpful to see whether the transformation takes the series in to Stationarity

The residuals from third degree polynomial trend stabilize the series with mean 0 and variance 1.96 . The third difference stabilizes the series with mean 0.24 and variance 9.2. We take this polynomial to remove the trend (i.e. to detrend) the series and to fit the mean part of the series. The residuals deviate from this mean fitted from the trend is time series variables to be used for ARMA

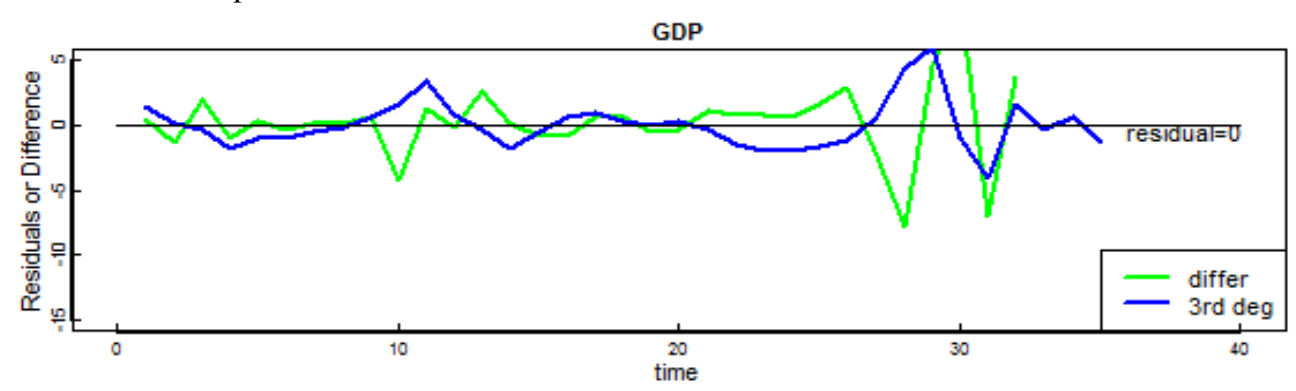

Figure 4. Differenced and detrended series of GDP. 
To identify the ARMA order of the series obtained from differencing and detrending we plot the ACF and PACF of the new series
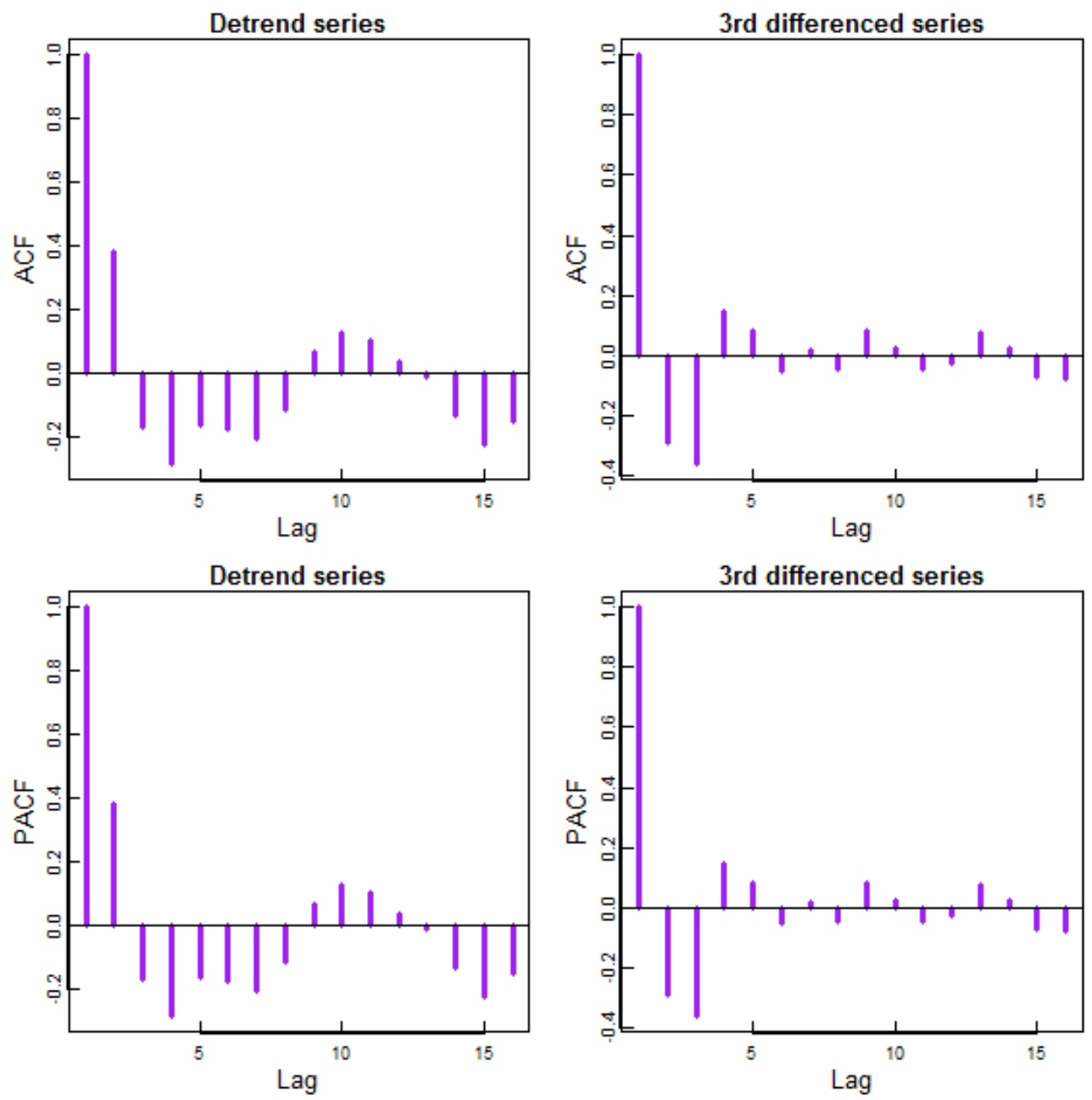

Figure 5. The autocorrelation and partial autocorrelation of the new series.

The plot in Figure 5 shows the ACF and PACF values drops down to zero except in the first few lags. Thus there are significant ACF and PACF in both series. This shows the series has autoregressive order and moving average orders.

Next we apply ARIMA model in all stationary series. We have transformed GDP (third differenced series), GDP growth rate and inflation rate. Our aim here is to determine the order of ARIMA which is best forecasting model. Besides the ACF and PACF plot we have powerful test, Portmanteau statistic, of order of ARIMA model using the residuals come out of this model. The statistic is such that if the model $\operatorname{ARMA}(p, q)$ is appropriate the residuals do not have correlation up to lag $m$ with $m-p-q$ df of chi-square distribution. We use $m=\log (n), \mathrm{m}=5$ wherever appropriate. If the null hypothesis is true we found larger $(>0.05) \mathrm{p}$-value.

We try to fit the ARIMA for GDP, GDP rate and inflation rate. We try to search for better model using AIC and BIC. Table 2 presents ARIMA of competing different orders and model selection tool AIC and BIC and Box-Ljung test of Portmanteau statistic.

There are various methods for selection of order of ARMA model. Sometimes we compare models by using mean square error. But this criterion does not consider the number of parameters involved in the model. More useful consideration is information criteria that consider the number of parameters in the model and the residual likelihood. We use AIC, BIC and variance in Table 2 as a model selection tools.

Table 2. Model selection by using Information criteria and Box-Ljung test.

\begin{tabular}{llllll}
\hline Time series & order $(p, d, q)$ & $\sigma^{2}$ & AIC & BIC & $\chi_{\alpha}^{2}(m-p-q)(\mathbf{p}$-val) \\
\hline \multirow{3}{*}{ GDP } & $1,0,0$ & NA & NA & NA & \\
& $1,2,0$ & 7.975 & 166.26 & 169.2562 & \\
& $1,2,1$ & 6.354 & 161.23 & 165.7212 & \\
& $1,2,2$ & 6.113 & 162.03 & 168.0114 & $0.95(0.329) d f=1, m=5$ \\
\hline
\end{tabular}




\begin{tabular}{|c|c|c|c|c|c|}
\hline Time series & $\operatorname{order}(p, d, q)$ & $\sigma^{2}$ & AIC & BIC & $\chi_{\alpha}^{2}(m-p-q)(\mathbf{p}-\mathbf{v a l})$ \\
\hline \multirow{4}{*}{ GDP rate } & $1,1,1$ & 42.03 & 224.07 & 228.5613 & \multirow{4}{*}{$4.713(0.0947), d f=2, m=6$} \\
\hline & $1,1,2$ & 35.54 & 220.85 & 226.838 & \\
\hline & $2,1,2$ & 32.32 & 219.93 & 227.411 & \\
\hline & $2,1,3$ & 31.09 & 220.75 & 229.7247 & \\
\hline \multirow{5}{*}{ Inflation rate } & $0,1,0$ & 186.8 & 268.24 & 269.7413 & \multirow{5}{*}{$1.5215,(0.2174), d f=1, m=3$} \\
\hline & $1,1,0$ & 153.5 & 263.95 & 266.9411 & \\
\hline & $1,1,1$ & 113.5 & 257.05 & 261.5429 & \\
\hline & $2,1,1$ & 112.9 & 258.46 & 264.4491 & \\
\hline & $1,1,2$ & 112.3 & 258.72 & 264.7015 & \\
\hline
\end{tabular}

Minimum MSE, AIC and BIC is corresponds to ARIMA $(2,2,2)$ for annual GDP. The Box-Ljungs test suggests this model is appropriate. The minimum MSE is corresponds to ARIMA $(2,1,3)$ for GDP rate. But the AIC and BIC of this model is a little beat larger than $\operatorname{ARIMA}(2,1,2)$. This is due to the additional parameter involved in the model. We used ARIMA $(2,1,2)$ of minimum AIC and BIC for inflation rate. We used these selected models

for forecasting the series.


Goodness of fit

To forecast these characteristics let us test the goodness of the model. If our model is good then the residuals of the model is white noise. If the model is good fit to the observed series the residuals bounded between $\pm 1.96 \sigma$ (as Figure 6 column 1) and the residuals do not correlate (as Figure 6 column 2), and the residuals are independently and identically distributed. In table 2 of the last column the pvalue shows the model is good.






Figure 6. Model Diagnostic through residual and autocorrelation and Box-Ljung test.

Standard residuals for all series are bounded between -3 and 3 which shows the fitted model is good. The ACF plot shows the residuals series in any lag do not have significant correlation this shows the residuals are white nois and independently distributed. The p-values in all lags are greater than our significance level which suggests to accept the hypothesis of the fitted order of the series $\operatorname{ARIMA}(p, d, q)$ is correct.

Forecasts

We forecast the three series variables for five years. Our model for GDP is $\operatorname{ARIMA}(2,2,2)$, for GDP growth rate is $\operatorname{ARIMA}(2,1,2)$ and for inflation rate is $\operatorname{ARIMA}(1,1,1)$. The following figure presents the future forecasts of the three series 2016-2025 
GDP

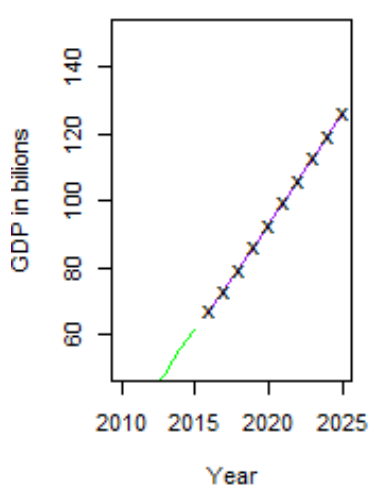

(a)
GDP rates



(b) inflation

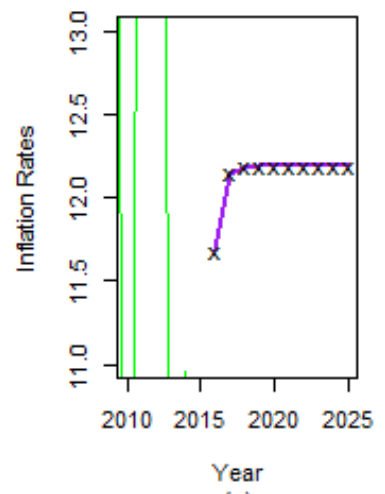

(c)

Figure 7. Forecasting series of GDP, GDP rate and inflation rate.

The GDP continue to its trend, The GDP growth rate for the coming years will be 9.99 in average and the inflation fluctuates a little beat. We take only the first few lags of the forecast as valid. Because as the forecast lag increases the variance of the forecast increases and makes it less precise. Table 3 presents the forecast of the series which is the same as Figure 7 above but only few first lags.

Table 3. Forecasts.

\begin{tabular}{lllll}
\hline Year & $\mathbf{2 0 1 6}$ & $\mathbf{2 0 1 7}$ & $\mathbf{2 0 1 8}$ & $\mathbf{2 0 1 9}$ \\
\hline GDP in billions of USD (estimated) & 67.094 & 73.013 & 79.397 & 86.083 \\
GDP rate (estimated) & 10.084 & 10.121 & 9.924 & 9.976 \\
Inflation rate (estimated) & 11.682 & 12.146 & 12.186 & 10.035 \\
\hline
\end{tabular}

NB: forecasts are less precise as the forecast lags increase.

Forecast table shows the GDP is increasing trend. The average of forecasts of GDP growth rate is 10.028 . We found very impressive result as we forecast the GDP growth rates using the forecast GDPs. If we use forecast GDPs to calculate GDP growth rates for 2016 what would happen? $(61.537-67.094) / 61.537 * 100=9.1$ this is very close to 10.084 . Closer values also obtained for other lags but decreasing precession.

\section{Conclusion}

The purpose of this research was to find suitable model to explain underlying series of annual GDP, GDP rates and Inflation rates of Ethiopia. We use Box-Jenkins methodology to see the behavior of these variables. The ARIMA model is used to forecast the future behavior of these series. The GDP is in line of increasing cubic trend and the GDP rate fluctuates and in the last decades it comes to increase, whereas the inflation rate fluctuates throughout the observation periods. We try to find the "best" ARIMA model using information criteria and Box-Jung's appropriate lag test and we found good predictive models. We found $\operatorname{ARIMA}(2,2,2)$ is appropriate for annual country GDP, and ARIMA $(2,1,2)$ for GDP rate and ARIMA $(1,1,1)$ for inflation rates. Using these models we forecast the the future values of the process. Annual country GDP continued growing in its trend and GDP rate continued to grow at an average rate of 10.028 . And the inflation showed constant trend. We use other method of forecasting GDP growth rates. The forecast GDPs were used to calculate GDP growth rates and we found impressive values which was very closer to the forecasts in ARIMA model.

\section{References}

[1] ANNUA 2012 report: United Nations development programe in Ethiopia, Transformation, excellence and action.

[2] Pasapitch Chujai, Nittaya Kerdprasop, and Kittisak Kerdprasop, Time series analysis of house hold electric consumption with ARIMA and ARMA models.

[3] Robinson Kruse; creates research paper 2010-28.

[4] Box, G. E. R., Jenkins, G. M.: Time Series Analysis, Forcasting and control, holden days Sanfransisco 1976.

[5] A. Ion. McLeod, Hao Yu, Esam Mahdi, 2011: Time series analysis with $\mathrm{R}$.

[6] Sources of Inflation and Economic Growth in Ethiopia Part II By Teshome A. (PhD) 01 September 2011.

[7] Jenkins, D. M., Watts, D. G.: Spectral Analysis and its application, holden days Sanfransisco 1968.

[8] Marea Fazekas 2004: Application of time series models on medical research.

[9] Barsky, R. B. (1987): "The Fisher hypothesis and the forecastibility and persistence of inflation journal of monitory economics 19, 3-24.

[10] Tewodros Gebru, 2015: Determinants of economic growth in Ethiopia, A time series analysis, Msc thesis, Addis Ababa University. 
[11] Javer Contreras et. al 2003: ARIMA models to predict next day electricity price, IEEE TRANSACTIONS ON POWER SYSTEMS, VOL. 18, NO. 3.

[12] Burdekin, R. C. K. and P. L. Siklos (1999): "Exchange rate regimes and shifts in inflation persistence: does nothing else matter Journal of Money, Credit and Banking 31, 235-247.

[13] Mankiw, N. G., J. A. Miron, and D. N. Weil (1987): "The adjustment of expectations to changek in regime: a study of the founding of the federal reserve." American Economic Review 77,

[14] Hakkio, C. S. and M. Rush (1991): "Is the budget deficit too large?" Economic Inquiry.

[15] Wayne A. Fuller 1996: Introduction to statistical time series, second Edition, Wiley Series in probability and statistics.

[16] Alexander Aue, 2010: Applied Time series analysis, Lecture notes, University of California, Davis.

[17] Robert Yaffee with Monnie McGee; 1999: Introduction to time series analysis and forecasting with application of SAS and SPSS.
[18] W. N. Venables, D. M. smith and the R-core team, 2016: An introduction to $\mathrm{R}$.

[19] Alemayehu Geda, Addis yimer, 2014: Growth, Poverity and Inquality in Ethiopia, 2000-2013: Amacroeconomic Appraisal, Department of Economics Addis Ababa university.

[20] Chinoso Michael, Oluchukuwu Chukuwuemeka, Charity Uchena, Nnaemeka Martin Eze, Chukwunenya Ifeanyi Okonkwo. On the Fourier Residual Modification of Arima Models in Modeling Malaria Incidence Rates among Pregnant Women. American Journal of Theoretical and Applied Statistics Vol. 9, No. 1, 2020, pp. 1-7. doi: 10.11648/j.ajtas.20200901.11

[21] Admasu Shiferaw, 2017: Productive capacity and Economic growth in Ethiopia. Department of Economic and social affairs. CDP Background Paper No. 34.

[22] Esubalew Asmare Shilea 2019: Economic Growth- Quality of Life Nexus in Ethiopia: Time Series Analysis.

[23] Adisie Melak 2018: The Contribution of Foreign Direct Investment for Economic growth of Ethiopia: Time Series Analysis DebreTabor University, Ethiopia 